\title{
Influência do Sistema de Criação sobre o Desempenho, a Condição Fisiológica e o Comportamento de Linhagens de Frangos para Corte
}

\author{
Marco Aurélio Neves da Silva1, Paulo Hellmeister Filho², Millor Fernandes do Rosário ${ }^{3}$, \\ Antonio Augusto Domingos Coelho ${ }^{4}$, Vicente José Maria Savino ${ }^{4}$, Antonio Augusto Franco \\ Garcia $^{4}$, Iran José Oliveira da Silva ${ }^{5}$, José Fernando Machado Menten ${ }^{6}$
}

\footnotetext{
RESUMO - Realizou-se o presente trabalho com o intuito de avaliar a influência do sistema de criação (intensivo e semi-intensivo) no desempenho (peso corporal e conversão alimentar), na condição fisiológica sob estresse térmico (temperatura retal, freqüência respiratória e hematócrito) e comportamento (freqüência ao pasto) de frangos de corte. Foram utilizadas quatro linhagens de frangos de corte, duas tipo caipira e duas comerciais. Para avaliação do desempenho e condição fisiológica das aves nas idades de $45,55,65$ e 75 dias, instalou-se um experimento no qual as aves foram alojadas em boxes constituídos de $4,5 \mathrm{~m}^{2}$ de área interna (abrigo) e $35 \mathrm{~m}^{2}$ de área de pastejo com lotação de 35 aves/box. Outro experimento foi instalado para avaliação da freqüência das aves ao pasto entre o $35^{\circ}$ e $75^{\circ}$ dia de idade. Uma das linhagens avaliadas não demonstrou ser adaptada ao sistema semi-intensivo de criação. Verificaram-se diferenças significativas nos parâmetros de desempenho e de condição fisiológica das aves nos dois sistemas. Na criação semi-intensiva obtiveramse menores valores para temperatura retal, taxa respiratória e hematócrito e melhores valores de peso corporal e conversão alimentar. Concluiu-se que a criação semi-intensiva proporcionou condições que aumentaram o bem-estar das aves, tendo influenciado positivamente o desempenho e a condição fisiológica das linhagens avaliadas, mesmo sob condições de estresse térmico.
}

Palavras-chave: adaptação, bem-estar, comportamento, sistema semi-intensivo

\section{Influence of the Rearing System on Performance, Physiological Condition and Behaviour of Broilers Lines}

\begin{abstract}
The objectives of this research were to evaluate the influence of rearing systems (intensive or semi-intensive) on the performance (body weight and feed efficiency), chicken physiological condition under heat stress (rectal temperature, respiratory frequency and hematocrit) and behavioural parameters (frequency in the pasture areas) of broilers lines. To evaluate broiler's performance and physiological condition on the ages of 45, 55, 65 and 75 days one experiment was carried out in boxes with 4,5 $\mathrm{m}^{2}$ of inside area and $35 \mathrm{~m}^{2}$ of outside area were 35 broilers were reared in each box. Another experiment was carried out to evaluate the frequency of broilers it the pasture areas from 35 to 75 days of age. One of the evaluated lines did not show adaptation to the semi-intensive rearing system. Significant differences were found in broilers performance and physiological conditions in both systems. In the semi-intensive rearing system the rectal temperature, respiratory frequency, hematocrit and feed efficiency were smaller and body weight larger than in the intensive rearing system. It was concluded that the semi-intensive rearing system provided conditions that increased broilers welfare and positively influenced the physiological conditions and performance of the broilers.
\end{abstract}

Key Words: adaptation, behaviour, semi-intensive system, welfare

\section{Introdução}

A grande procura dos consumidores por produtos diferenciados e de qualidade superior vem influenciando mudanças nos sistemas utilizados para produção de frangos (Gessulli, 1999; Vercoe et al., 2000). A sociedade está interessada em sistemas de produção que aumentem o bem-estar na criação de animais
(Verbeke \& Viane, 2000; Von Borell \& Van Den Weghe, 1999) e a implementação de mudanças que melhorem o bem-estar animal pode garantir a oferta desses novos produtos para os consumidores (Blokhuis et al., 2000; Fraser, 2001).

O regime de confinamento causa estresse intenso (Jones \& Mills, 1999), tendo como conseqüência respostas fisiológicas e comportamentais (Marin et

\footnotetext{
1 Bolsista CAPES (DR) - Departamento de Genética - ESALQ/USP. E.mail: mansilva@esalq.usp.br - Av. Pádua Dias, 11. Cp. 83. CEP: $13470-900$ - Piracicaba/SP/Brasil.

2 Docente - Departamento de Ciências Agrárias e Ambientais - Universidade Estadual de Santa Cruz - Ilhéus/BA.

3 Bolsista CAPES (MS) - Departamento de Genética - ESALQ/USP.

4 Docente - Departamento de Genética - ESALQ/USP.

5 Docente - Departamento de Engenharia Rural - ESALQ/USP.

6 Docente - Departamento de Produção Animal - ESALQ/USP.
} 
a1., 2001) que podem causar sérios problemas à saúde e bem-estar dos animais (Abeyesinghe et al., 2001; Hall, 2001; Mendl, 1999). Por estes motivos o sistema de criação semi-intensivo é considerado como uma alternativa. Este sistema, informalmente conhecido como "tipo caipira", permite que as aves tenham livre acesso às áreas de pastejo, resultando em diferenças particulares na qualidade da carne das mesmas quando comparada com a das aves criadas confinadas.

Conforme Silva \& Nakano (1998), essas diferenças ocorrem devido principalmente a ingestão pela ave de pasto, verduras, insetos, larvas, minhocas etc., que são abundantes no sistema semi-intensivo de criação. Sendo assim, consumidores mais tradicionais preferem a carne de aves criadas semi-confinadas por possuir um sabor mais "natural" do que a carne de aves criadas totalmente confinadas.

O bem-estar do animal e sua saúde devem ser considerados em um sistema de criação (Bockisch et al., 1999). Segundo Blokhuis et al. (2000), produção e qualidade estão ligadas ao bem-estar do animal, portanto, os sistemas de criação devem evoluir para atender as necessidades dos animais e dos consumidores (Verbeke \& Viane, 2000 e Vercoe et al., 2000).

As condições ambientais podem influenciar a produção (Silva \& Silva, 1998), o comportamento (Dawkins, 1999) e a condição fisiológica dos animais (Furlan et al., 1999). Estudos que vêm sendo realizados na área de genética tentam desenvolver aves com melhor adaptabilidade, visando a melhora dos índices zootécnicos da criação (Ferrante et al., 2001; Marin et al., 2001; Silva et al., 2001).

Objetivou-se neste trabalho avaliar a influência do sistema de criação (intensivo e semi-intensivo) no desempenho (peso corporal e conversão alimentar), na condição fisiológica sob estresse térmico (temperatura retal, taxa respiratória e hematócrito) e comportamento (freqüência ao pasto) de frangos de corte.

\section{Material e Métodos}

No primeiro experimento, foram utilizadas quatro linhagens de frangos para corte, duas tipo caipira, Caipirinha de crescimento lento e 7P de crescimento rápido e duas comerciais, $\mathrm{C} 1$ de crescimento lento e $\mathrm{C} 2$ de crescimento rápido.

Os pintos de um dia, obtidos no incubatório experimental do Departamento de Genética - ESALQ/ USP, foram vacinados contra a doença de Marek e a seguir alojados sem separação de sexo em 8 boxes experimentais, quatro no sistema intensivo e quatro no sistema semi-intensivo, sendo um box por linhagem em cada sistema de criação com uma lotação de 35 pintos/box. Cada box possuía $4,5 \mathrm{~m}^{2}$ de área interna, sendo que os boxes do sistema semi-intensivo tinham acesso à área para pastejo com $35 \mathrm{~m}^{2}$.

Na primeira semana foram utilizadas lâmpadas incandescentes (150 Watts) para aquecimento das aves, uma para cada box. Até o sétimo dia foram utilizados bebedouros do tipo pressão e comedouros tubulares infantis na proporção de dois equipamentos de cada tipo por box. A partir do oitavo dia, a água passou a ser fornecida em bebedouros do tipo pendular e a ração em comedouros tubulares para aves adultas, na quantidade de um equipamento de cada tipo por box. Ração e água foram distribuídas à vontade para as aves durante todo o período de criação que se estendeu até 75 dias de idade. As aves receberam vacinação contra as doenças de Newcastle e Gumboro na água de bebida, aos 7 e 21 dias de idade, conforme recomendação do fabricante das vacinas.

Quando as aves atingiram 21 dias de idade, o acesso ao pasto dos boxes do sistema semi-intensivo foi aberto, permitindo a livre passagem das aves desses boxes para os respectivos pastos. O período de coleta de dados desenvolveu-se entre 45 e 75 dias de idade, utilizando-se as idades de 45, 55, 65 e 75 dias para monitoramento do peso corporal, temperatura retal, freqüência respiratória e hematócrito das aves. Todos os parâmetros foram obtidos, escolhendo-se ao acaso 5 machos de cada box, quando a temperatura e umidade relativa no box e no pasto eram de $32^{\circ} \mathrm{C}$ e $46 \%$, respectivamente. Vale ressaltar que esta condição ambiental foi escolhida com o intuito de se avaliar as linhagens em condições de estresse térmico.

O peso corporal foi obtido através de pesagem em balança tipo dinamômetro com capacidade até $5 \mathrm{~kg}$ e precisão de $20 \mathrm{~g}$ e a conversão alimentar obtida a partir da relação entre o consumo de ração e o peso corporal no período experimental. Os índices de temperatura retal foram obtidos de acordo com Silva et al. (2001), introduzindo-se o sensor de um teletermômetro (Max-Min Thermo Hygro) na cloaca das aves. A freqüência respiratória foi obtida através de avaliação visual levando-se em consideração o número de vezes que as aves inspiraram ar por minuto de tempo. O hematócrito foi determinado através da técnica do micro-hematócrito, descrita por Rosário et al. (2000), coletando-se amostras de sangue das aves em microtubos capilares e a seguir

R. Bras. Zootec., v.32, n.1, p.208-213, 2003 
centrifugando-se os mesmos a $12.000 \mathrm{x} \mathrm{g,.} \mathrm{A} \mathrm{deter-}$ minação dos valores de hematócrito foi feita através de leitura com o auxílio de cartão de leitura (FANEM Ltda) com escalas padronizadas.

O delineamento experimental foi em faixa com parcelas subdivididas sendo as linhagens alocadas nas parcelas e o sistema de criação (intensivo ou semi-intensivo) nas sub-parcelas. A análise de variância dos parâmetros avaliados foi processada pelo programa de computação SAS (1985) no procedimento GLM (General Linear Models), e a comparação de médias foi realizada utilizando-se o teste de Tukey. O modelo matemático correspondente foi:

$$
\begin{gathered}
Y i j k=u+L i+e i+S j+e j+(L \times S) i j+e i j+I k+ \\
(I \times L) i k+e i j k
\end{gathered}
$$

em que: Yijk = valor observado da ave da linhagem i, no sistema de criação $\mathrm{j}$, na idade $\mathrm{k} ; \mathrm{u}=$ média geral; $\mathrm{Li}=$ efeito da linhagem $\mathrm{i}$; ei = erro a (aleatório); $\mathrm{Sj}=$ efeito do sistema de criação j; ej = erro b (aleatório); (L x S)ij = efeito da interação da linhagem i com o sistema de criação $\mathrm{j}$; eij = erro c (aleatório); $\mathrm{Ik}=$ efeito da idade k; (I x L)ik = efeito da interação da idade $\mathrm{k}$ com a linhagem i; eijk = erro d (aleatório).

No segundo experimento, realizado para avaliação da freqüência das aves ao pasto, foram utilizadas as quatro linhagens citadas na descrição do primeiro experimento e os pintos de um dia, obtidos no incubatório experimental do Departamento de Genética - ESALQ/USP, foram vacinados ao nascer contra a doença de Marek e alojados sem separação de sexo em 16 boxes experimentais no sistema semiintensivo de criação, sendo 4 boxes por linhagem. Cada box possuía $4,5 \mathrm{~m}^{2}$ de área interna com acesso a área de pastejo de $35 \mathrm{~m}^{2}$ com uma lotação de 35 pintos/box. O manejo das aves foi realizado como descrito no primeiro experimento e o período experimental se estendeu até 75 dias de idade.

Quando as aves atingiram 21 dias de idade, o acesso ao pasto dos boxes foi aberto, permitindo a livre passagem das aves de cada box para o respectivo pasto. Após um período de 14 dias de adaptação, iniciou-se o período de monitoramento que se desenvolveu entre 35 e 75 dias de idade, durante o qual foram coletados dados de duas em duas horas, a partir de 8 até 20 h (horário de Brasília), da quantidade de aves presentes no pasto. A freqüência ao pasto das linhagens foi obtida a partir das médias diárias da quantidade de aves no pasto.

O delineamento experimental foi em blocos casualizados com quatro repetições, sendo cada bloco composto por quatro boxes, um para cada linhagem. A análise de variância e a comparação de médias foram realizadas utilizando-se os mesmos procedimentos citados na descrição do experimento anterior. O modelo matemático correspondente foi:

$$
Y i j=u+L i+B j+e i j
$$

em que: Yij = valor observado da linhagem $\mathrm{i} ; \mathrm{u}=$ média geral; $\mathrm{Li}=$ efeito da linhagem $\mathrm{i} ; \mathrm{Bj}=$ efeito do bloco j; eij = erro (aleatório).

\section{Resultados e Discussão}

A comparação de médias entre as quatro linhagens para os parâmetros de desempenho (peso corporal e conversão alimentar) e de condição fisiológica (temperatura retal, freqüência respiratória e hematócrito) está apresentada na Tabela 1.

A linhagem $\mathrm{C} 2$ apresentou maiores valores para todos os parâmetros avaliados, enquanto que a linhagem $\mathrm{C} 1$ apresentou os menores valores, com exceção para o parâmetro conversão alimentar. Foram detectadas diferenças significativas entre as quatro linhagens para todos os parâmetros avaliados, com exceção das comparações entre as linhagens C2 e 7P para o parâmetro peso corporal e entre a linhagem $\mathrm{C} 2$ e as demais para o parâmetro conversão alimentar.

Segundo Eberhart \& Washburn (1993), Mazzi (1998) e Yahav et al. (1998), aves que apresentam maiores índices de temperatura retal e de freqüência respiratória em condições de estresse térmico são menos resistentes ao calor. Assim, a linhagem C2 que apresentou temperatura retal e freqüência respiratória superiores as das outras três linhagens pode ser considerada menos resistente ao calor.

Os valores de hematócrito apresentados pelas linhagens são considerados em faixa intermediária por Maxwell etal.(1992), não indicando suscetibilidade das linhagens a síndrome ascítica. Segundo Shlosberg et al. (1992), aves com valores de hematócrito elevados apresentam maior suscetibilidade a síndrome ascítica, portanto, a utilização de linhagens que apresentem baixos valores de hematócrito e de um sistema de criação adequado podem minimizar as conseqüências dessa síndrome.

Na comparação entre as médias dos parâmetros 
Tabela 1 - Comparação das linhagens para peso corporal (PC), conversão alimentar (CA), temperatura retal (TR), freqüência respiratória $(\mathrm{FR})$ e hematócrito $(\mathrm{H})$

Table 1 - Lines comparison for body weight (BW), feed efficiency (FE), rectal temperature (RT), respiratory frequency (RF) and hematocrit $(H)$

\begin{tabular}{lccccc}
\hline Linhagens & $\mathrm{PC}(\mathrm{g})$ & $\mathrm{CA}(\mathrm{g} / \mathrm{g})$ & $\mathrm{TR}\left({ }^{\circ} \mathrm{C}\right)$ & FR $($ resp.min & \\
Lines & $B W(\mathrm{~g})$ & $F E(g / g)$ & $R T\left({ }^{-1}\right)$ & $\left.R F(\text { resp.min })^{-1}\right)$ & $\mathrm{H}(\%)$ \\
\hline $\mathrm{C} 2$ & $2490,00^{\mathrm{a}}$ & $3,10^{\mathrm{a}}$ & $42,23^{\mathrm{a}}$ & $115,10^{\mathrm{a}}$ & $31,11^{\mathrm{a}}$ \\
7P & $2411,75^{\mathrm{a}}$ & $2,92^{\mathrm{b}}$ & $41,99^{\mathrm{b}}$ & $112,40^{\mathrm{b}}$ & $30,59^{\mathrm{b}}$ \\
Caipirinha & $1895,50^{\mathrm{b}}$ & $2,91^{\mathrm{b}}$ & $41,95^{\mathrm{c}}$ & $99,70^{\mathrm{c}}$ & $29,91^{\mathrm{c}}$ \\
$\mathrm{Cl}$ & $1662,50^{\mathrm{c}}$ & $2,94^{\mathrm{b}}$ & $41,75^{\mathrm{d}}$ & $94,05^{\mathrm{d}}$ & $29,09^{\mathrm{d}}$ \\
\hline
\end{tabular}

Médias seguidas de letras diferentes na mesma coluna diferem pelo teste de Tukey $(P<0,01)$.

Means, whithin a column, followed by different letters are different by Tukey test $(P<.01)$.

avaliados, verificadas nos dois sistemas de criação (Tabela 2), foi verificada diferença significativa entre os sistemas, sendo que se observou maior valor de peso corporal e menores valores de temperatura retal, taxa respiratória e hematócrito em aves criadas no sistema semi-intensivo, demonstrando a influência positiva do sistema de criação nesses parâmetros e consequentemente no conforto e bem-estar das aves.

A idade influenciou os valores dos parâmetros avaliados (Tabela 3 ) ou seja, quanto mais velhas as aves, maiores valores de peso corporal, temperatura retal, freqüência respiratória e hematócrito foram apresentados resultando em diferenças significativas entre as idades para os parâmetros. Esses resultados confirmam os encontrados por Rosário et al. (2000) e Silva et al. (2001).

Na Tabela 4 estão apresentados os valores de freqüência ao pasto das quatro linhagens avaliadas. A linhagem C2 apresentou menor freqüência ao pasto (6,6 aves/dia) durante o período experimental, diferindo estatisticamente das outras três linhagens, sendo que a linhagem $\mathrm{C} 1$ foi a que apresentou maior freqüência ao pasto $(9,6$ aves/dia).
Segundo Craig \& Muir (1996) e Ferrante et al. (2001), o comportamento do animal está ligado ao ambiente de criação e segundo Jones et al. (2000) e Von Borell \& Van Den Weghe (1999) a melhora deste ambiente pode beneficiar a criação. A freqüência ao pasto das aves ou seja, o comportamento das aves, foi afetado pelos fatores ambientais avaliados, demonstrando a importância do estudo dos fatores ambientais que podem além de afetar o comportamento afetar também o bem-estar dos animais.

Duncan (1998) cita que o comportamento pode ser utilizado como parâmetro para avaliar a qualidade do bem-estar das aves; portanto, uma vez que o sistema semi-intensivo de criação proporcionou condições mais adequadas ao comportamento natural das aves, pode-se concluir que esse sistema contribuiu para a melhora do bem-estar das linhagens.

De acordo com Sundrum (2001), o aumento da área de locomoção do animal pode afetar sua saúde e seu bem-estar positivamente. Portanto a criação de aves utilizando o sistema semi-intensivo permite que as aves permaneçam no pasto, se locomovam mais e tenham seu bem-estar melhorado.

Tabela 2 - Comparação dos sistemas de criação para peso corporal (PC), conversão alimentar (CA), temperatura retal (TR), freqüência respiratória (FR) e hematócrito $(H)$

Table 2 - Rearing systems comparison for body weight (BW), feed efficiency (FE), rectal temperature (RT), respiratory frequency (RF) and hematocrit (H)

\begin{tabular}{lccccc}
\hline $\begin{array}{l}\text { Sistemas } \\
\text { Systems }\end{array}$ & ${ }^{1} \mathrm{PC}(\mathrm{g})$ & ${ }^{2} \mathrm{CA}(\mathrm{g} / \mathrm{g})$ & ${ }^{2} \mathrm{TR}\left({ }^{\circ} \mathrm{C}\right)$ & ${ }^{2} \mathrm{FR}\left(\mathrm{resp} \cdot \mathrm{min}^{-1}\right)$ & ${ }^{2} \mathrm{H}(\%)$ \\
$R F\left(\operatorname{resp} \cdot \mathrm{min}^{-1}\right)$ & $\left.H^{\circ}\right)$ \\
\hline $\begin{array}{l}\text { Intensivo } \\
\begin{array}{l}\text { Intensive } \\
\text { Semi-intensivo }\end{array}\end{array}$ & $2070,38^{\mathrm{a}}$ & $3,13^{\mathrm{a}}$ & $42,10^{\mathrm{a}}$ & $106,82^{\mathrm{a}}$ & $30,57^{\mathrm{a}}$ \\
$\begin{array}{l}\text { Semi-intensive } \\
\text { Sem }\end{array}$ & $2159,50^{\mathrm{b}}$ & $2,80^{\mathrm{a}}$ & $41,86^{\mathrm{b}}$ & $103,80^{\mathrm{b}}$ & $29,82^{\mathrm{b}}$
\end{tabular}

Médias seguidas de letras diferentes na mesma coluna diferem pelo teste de Tukey $\left({ }^{1} \mathrm{P}<0,05\right.$ e $\left.{ }^{2} \mathrm{P}<0,01\right)$.

Means, whithin a column, followed by different letters are different by Tukey test $\left({ }^{1} P<.05\right.$ and $\left.{ }^{2} P<.01\right)$.

R. Bras. Zootec., v.32, n.1, p.208-213, 2003 
Tabela 3 - Comparação das idades para peso corporal (PC), conversão alimentar (CA), temperatura retal (TR), freqüência respiratória $(F R)$ e hematócrito $(H)$

Table 3 - Ages comparison for body weight (BW), feed efficiency (FE), rectal temperature (RT), respiratory frequency (RF) and hematocrit $(H)$

\begin{tabular}{|c|c|c|c|c|c|}
\hline $\begin{array}{l}\text { Idade (dias) } \\
\text { Ages (days) }\end{array}$ & $\begin{array}{l}\mathrm{PC}(\mathrm{g}) \\
B W(g)\end{array}$ & $\begin{array}{l}\mathrm{CA}(\mathrm{g} / \mathrm{g}) \\
F E(g / g)\end{array}$ & $\begin{array}{l}\operatorname{TR}\left({ }^{\circ} \mathrm{C}\right) \\
R T\left({ }^{\circ} \mathrm{C}\right)\end{array}$ & $\begin{array}{l}\mathrm{FR}\left(\text { resp. } \mathrm{min}^{-1}\right) \\
R F(\text { resp.min }\end{array}$ & $\begin{array}{l}\mathrm{H}(\%) \\
H(\%)\end{array}$ \\
\hline 75 & $2753,00^{\mathrm{a}}$ & $3,19^{a}$ & $42,11^{\mathrm{a}}$ & $110,00^{\mathrm{a}}$ & $30,89^{a}$ \\
\hline 65 & $2355,25^{\mathrm{b}}$ & $2,95^{\mathrm{b}}$ & $42,04^{\mathrm{b}}$ & $106,50^{b}$ & $30,31^{b}$ \\
\hline 55 & $1871,00^{\mathrm{c}}$ & $2,42^{c}$ & $41,98^{\mathrm{c}}$ & $103,80^{c}$ & $29,91^{b c}$ \\
\hline 45 & $1480,50^{\mathrm{d}}$ & $2,32^{\mathrm{c}}$ & $41,78^{d}$ & $100,90^{\mathrm{d}}$ & $29,60^{c}$ \\
\hline
\end{tabular}

Médias seguidas de letras diferentes na mesma coluna diferem pelo teste Tukey $(\mathrm{P}<0,01)$.

Means, whithin, a column followed by different letters are different by Tukey test $(P<.01)$.

Tabela 4 - Comparação das linhagens para freqüência ao pasto

Table 4 - Lines comparison for frequency in the pasture areas

$\left.\begin{array}{lc}\hline \begin{array}{l}\text { Linhagens } \\ \text { Lines }\end{array} & \begin{array}{c}\text { Freqüência ao pasto } \\ \left(\mathrm{n}^{\mathrm{o}} \text { de aves.dia }\right.\end{array} \\ \begin{array}{c}\text { Frequency in the pasture } \\ \text { areas (broilers number.day }\end{array}\end{array}\right)$

Médias seguidas de diferentes letras diferem pelo teste Tukey $(\mathrm{P}<0,05)$.

Means followed by different letters are different by Tukey test $(P<.05)$.

\section{Conclusões}

O sistema semi-intensivo de criação proporcionou condições que aumentaram o bem-estar das aves, influenciando positivamente o desempenho, condição fisiológica e comportamento das linhagens, mesmo sob condições de estresse térmico. A linhagem $\mathrm{C} 2$ demonstrou ser a menos adaptada ao sistema semi-intensivo entre as linhagens avaliadas.

\section{Literatura Citada}

ABEYESINGHE, S.M.; WATHES, C.M.; NICOL, C.J. et al. The aversion of broiler chickens to concurrent vibration and thermal stressors. Applied Animal Behaviour Science, v.73, n.3, p.199-215, 2001.

BLOKHUIS, H.J.; HOPSTER, H.; GEVERINK, N.A. et al. Studies of stresse in farm animals. Comparative Haematology International, v.8, n.2, p. 94-101, 1998.

BOCKISCH, F.J.; JUNGBLUTH, T.; RUDOVSKY, A. Technical indicators for evaluation of housing systems for cattle, pigs and laying hens relating to animal welfare. Zuchtungskunde, v.71, n.1, p.38-63, 1999.

CRAIG, J.V.; MUIR, W.M. Group selection for adaptation to multiple-hen cages: behaviour responses. Poultry Science, v.75, n.10, p.1145-1155, 1996.

DAWKINS. M.S. The role of behaviour in the assessment of poultry welfare. World's Poultry Science Journal, v.55, v.3, p.295-303, 1999.

DUNCAN, I.J.H. Behavior and behavioral needs. Poultry Science, v.77, n.12, p.1766-1772, 1998.

EBERHART, D.E.; WASHBURN, K.W. Variation in bodytemperature response of naked neck and normally feathered chickens to heat stress. Poultry Science, v.72, n.8, p.13851390, 1993.

FERRANTE, V.; VERGA, M.; MANGIAGALLI, M.G. et al. Behaviour reactions, semen quality and testosterone levels in cocks: genetic implications. Animal Welfare, v.10, n.3, p.269-279, 2001.

FRASER, D. The "new perception" of animal agriculture: legless cows, featherless chickens and a need for genuine analysis. Journal of Animal Science, v.79, n.3, p.634-641, 2001.

FURLAN, R.L.; MACARI, M.; MORAES, V.M.B. et al. Alterações hematológicas e gasométricas em diferentes linhagens de frangos de corte submetidos ao estresse calórico agudo. Revista Brasileira de Ciência Avícola, v.1, n.1, p.77-84, 1999.

GESSULLI, O.P. Avicultura alternativa: sistema "ecologicamente correto" que busca o bem-estar animal e a qualidade do produto final. Porto Feliz: OPG Editores, 1999. $217 \mathrm{p}$.

HALL, A.H. The effect of stocking density on the welfare and behaviour of broiler chickens reared commercially. Animal Welfare, v.10, n.1, p.23-40, 2001.

JONES, R.B.; CARMICHAEL, N.L.; RAYNER, E. Pecking preferences and pre-dispositions in domestic chicks: implications for the development of environmental enrichment devices. Applied Animal Behaviour Science, v.69, n.4, p.291-213, 2000.

JONES, R.B.; MILLS, A.D. Divergent selection for social reinstatement behavior in Japanese quail: effects on sociality and social discrimination. Poultry Avian Biology Review, v.10, n.4, p.213-223, 1999.

MAXWELL, M.H.; ROBERTSON, G.W.; McCORQUODALE, C.C. Whole blood and plasma viscosity values in normal and 
ascitic broiler chickens. British Poultry Science, v.33, v.4, p.871-877, 1992.

MAZZI, C.M. Análise da expressão da proteína de estresse Hsp70 em frangos de corte portadores do gene "Naked Neck" (pescoço pelado) submetidos a estresse térmico gradativo. Jaboticabal: Universidade Estadual Paulista, 1998. 60p. Dissertação (Mestrado em Agronomia) - Universidade Estadual Paulista, 1998.

MARIN, R.H.; FREYTES, P.; GUZMAN, D. et al. Effects of an acute stressor on fear and on the social reinstatement responses of domestic chicks to cagemates and strangers. Applied Animal Behaviour Science, v.71, n.1, p.57-66, 2001.

MENDL, M. Performing under pressure: stress and cognitive function. Applied Animal Behaviour Science, v.65, n.3, p.221-244, 1999.

ROSÁRIO, M.F.; SILVA, M.A.N.; MARTINS, E. et al. Influência do genótipo e do sexo sobre o valor hematócrito em galinhas reprodutoras pesadas. Revista Brasileira de Ciência Avícola, v.2, n.3, p.281-286, 2000.

SAS INSTITUTE. SAS user's guide: statistics. Raleigh: 1985. 956p.

SHLOSBERG, A.; ZADIKOV, I.; BENDHEIM, U. et al. The effects of poor ventilation, low tempertures, type of feed and sex of bird on the development of ascites in broilers: physiological factors. Avian Pathology, v.21, n.3, p.369-382, 1992.

SILVA, I.J.O; SILVA, M.A.N. Dicas de sucesso: fique por dentro de algumas medidas simples, voltadas à climatização da produção de frangos, que podem garantir o sucesso da criação neste verão. Avicultura Industrial, v.88, n.1059, p.46-47, 1998.
SILVA, M.A.N.; SILVA, I.J.O.; PIEDADE, S.M.S. et al. Resistência ao estresse calórico em frangos de corte de pescoço pelado. Revista Brasileira de Ciência Avícola, v.3, n.1, p.27-33, 2001.

SILVA, R.D.M.; NAKANO, M. Sistema Caipira de criação de galinhas. Piracicaba: O Editor, 1998. 110p.

SUNDRUM, A. Organic livestock farming - a critical review. Livestock Production Science, v.67, n.3, p.207-215, 2001.

VERBEKE, W.A.J.; VIANE, J. Ethical challenges for livestock production: meeting consumer concerns about meat safety and animal welfare. Journal of Agricultural \& Environmental Ethics, v.12, n.2, p.141-151, 2000.

VERCOE, J.E.; FITZHUGH, H.A.; Von KAUFMANN, R. Livestock productions systems beyond. Asian-Australian Journal of Animal Sciences, v.13, p.411-419, 2000.

VON BORELL, E.; VAN DEN WEGHE, S. Development of criteria for the assessment of housing systems for cattle, pigs and laying hens relating to animal welfare and environmental impact. Zuchtungskunde, v.71, n.1, p.8-16, 1999.

YAHAV, V.S.; LUGER, D.; CAHANER, A. et al. Thermoregulation in Naked Neck chickens subjected to different ambient temperatures. British Poultry Science, v.39, n.1, p.133-138, 1998.

Recebido em: $31 / 10 / 01$

Aceito em: 16/07/02 